\title{
On the Theory of a Participatory Firm
}

\author{
JAN SVEJNAR* \\ Department of Economics, Cornell University, \\ Ithaca, New York 14853
}

Received March 26, 1979; revised March 2, 1981

\section{INTRODUCTION}

Firms in which several different actors (or groups of actors) ${ }^{1}$ jointly determine company policy have come into existence in an increasing number of countries. ${ }^{2}$ Yet, with the exception of Steinherr's (1977) model, the existing theories of the firm generally do not reflect the institutional framework of a participatory firm. This paper presents two theoretical models of such a firm. The approach is quite general and nests the established theories of the firm as limiting cases.

Employee participation in management has been instituted primarily by law and in countries with a strong trade union movement. ${ }^{3}$ For this reason, possible influences of different systems of labor relations and trade union structures on the behavior of a participatory firm need to be explored. In this context it is shown that two different realistic assumptions about the objective functions of the relevant actors (or groups of actors) lead to strikingly different results in terms of the social efficiency of factor allocation. This finding is significant not only for the theory of a participatory firm but also for the theoretically limiting cases of a fully labor-managed firm or a traditional capitalist firm under collective bargaining.

* I have greatly benefited from discussions with Orley Ashenfelter, Gerald Epstein, Edward Green, Arvind Panagariya, Albert Rees, Jaroslav Vanek. and Henry Wan Jr. I have also received valuable comments from an anonymous referee and an associate editor of this journal. Any errors are, of course, my own.

${ }^{1}$ Most frequently these groups are the managers and the representatives of employees and shareholders, respectively.

${ }^{2}$ It is impossible to enumerate all the cases, but note that in Western Europe alone, Austria, Denmark, Germany, Netherlands, Norway, and Sweden have all established participatory firms by law. For details, see Schregle (1976) and Windmuller (1977).

${ }^{3}$ In fact, in most Western European countries the unions were instrumental in the establishment of a participatory system. Note also that the trade union movement is quite influential even in the fully labor-managed economy of Yugoslavia. 


\section{The Framework for ANAlyzing a PARTicipatory Firm}

In analyzing the behavior of a participatory firm, it is convenient to use as a framework the variable-bargaining-power model, ${ }^{4}$ which enables one to examine an entire spectrum of possible participatory systems. Briefly, the model generalizes the Nash $(1950,1953)$ solution to the two-party bargaining game. In the case of two parties, the objective function of the firm reflects the joint behavior of the two parties who act as if maximizing

$$
U=U_{1}^{\gamma_{1}} \cdot U_{2}^{\gamma_{2}}
$$

where $U_{1}$ and $U_{2}$ are the von Neumann-Morgenstern incremental utility functions of the two parties, $\gamma_{1}$ and $\gamma_{2}$ are their relative powers (degrees of control over the firm's policy), $\gamma_{1} \geqslant 0, \gamma_{2} \geqslant 0$, and $\gamma_{1}+\gamma_{2}=1 .{ }^{5}$ In the case where $n(n>2)$ parties participate in the decision-making the parties act as if they maximize:

$$
U=\prod_{i=1}^{n} U_{i}^{\gamma_{i}}
$$

with $U_{i}$ being the von Neumann-Morgenstern incremental utility function of party $i, \gamma_{i}$ being its relative power, $0 \leqslant \gamma_{i} \leqslant 1$ for all $i$, and $\sum_{i=1}^{n} \gamma_{i}=1$. The decision-making behavior of the firm can then be perceived as the search for a solution along an incremental utility frontier in $\mathbb{R}^{2}$ or $\mathbb{R}^{n}$, respectively. At the solution, the elasticity of substitution of $U_{i}$ for $U_{j}$ along the incremental utility frontier is equal to the relative power of parties $i$ and $j\left(\gamma_{i} / \gamma_{j}\right)$. As a result, the optimal values of $U_{i}$ and $U_{j}$ are proportional to the relative power of $i$ and $j$, adjusted for possible nonlinearity (strict concavity) of the incremental utility frontier:

$$
\frac{U_{i}^{*}}{U_{j}^{*}}=\frac{\gamma_{i}}{\gamma_{j}}\left(-\frac{\partial U_{i}}{\partial U_{j}}\right)^{6}
$$

In the usual Western European and, more recently, American system of participation by labor, management and shareholders, the three parties can be seen to act as if maximizing

$$
U=U_{L}^{\gamma_{L}} \cdot U_{M}^{\gamma_{M}} \cdot U_{S}^{\gamma_{S}}
$$

${ }^{4}$ See Svejnar (1977, 1980), Kalai (1977), and Roth (1979).

${ }^{5}$ When dealing with relative powers it is convenient to normalize by setting $\gamma_{1}+\gamma_{2}=1$. The von Neumann-Morgenstern utility functions are incremental in the sense that the threat point utility is zero.

${ }^{6}$ If the utility functions are linear $U_{i}^{*} / U_{j}^{*}=\gamma_{i} / \gamma_{j}$. 
where $U_{L}, U_{M}$, and $U_{S}$ are the respective incremental utility functions of labor, management, and shareholders, ${ }^{7}$ while $\gamma_{L}, \gamma_{M}$, and $\gamma_{S}$ are their corresponding degrees of power. Again, $0 \leqslant \gamma_{L}, \gamma_{M}, \gamma_{S} \leqslant 1$ and $\gamma_{L}+\gamma_{M}+\gamma_{S}=1$. While labor, management, and shareholders are the parties that most frequently codetermine the company policy in the existing participatory schemes, the present framework is quite general and can be used to analyze participatory systems which involve additional parties such as the representatives of the community in which the firm is located, the state, or foreign interests. Similarly, in situations where the management and shareholders are thought to have identical objectives $\left(U_{M}=U_{S}\right)$, Eq. (4) becomes $U=U_{L}^{\gamma_{L}} \cdot U_{M}^{y_{M}+\gamma_{S}}$ and the task is reduced to analyzing a two-party variable-power game characterized by (1).

Sections III and IV present two models which are both based on (4) but differ in the specification of the utility function, according to which of the alternative institutional systems is modelled. In both models the firm is assumed to produce a vector of $k$ outputs $Q_{1}, \ldots, Q_{k}$, using as inputs labor $L$, managerial input $M$, capital $K$, and a vector $X_{1}, \ldots, X_{n}$ of other inputs. Production possibilities are assumed to be strictly convex and the production function $f\left(Q_{1}, \ldots, Q_{k}, L, M, K, X_{1}, \ldots, X_{n}\right) \leqslant 0$ is also assumed to have continuous second derivatives. Together these conditions imply that the firstorder conditions for the maximizations performed in Sections III and IV are necessary and sufficient. The results of Section III and IV are discussed in Section V.

\section{MODEL I}

In Model I each party is assumed to use its power to maximize the unit price of the input it supplies, irrespective of the employment level of that input. This scenario corresponds to the objective function of a "pure" labormanaged firm as it was used by Ward (1958), Domar (1966), Vanek (1970), and others. In particular, in Model I the constituency of the bargainers are those workers, managers, and shareholders who remain with the firm once equilibrium is reached. As a result, in terms of the objective functions of the bargainers, it is only the expected per capita income of these workers, managers, and shareholders that counts. The income of others is irrelevant.

From an institutional standpoint, Model I is especially pertinent for analyzing participatory firms where the parties involved are organizations which operate only within the firm in question and where the input supply

${ }^{7}$ It is assumed that each party is homogeneous. This appears to be a reasonable assumption because intra-group conflicts of interest are likely to be minimized in the situations of bargaining with antagonistic groups. 
decisions within each party are made at the median or top of a clearly defined hierarchy. These parties are likely to behave in the spirit of Model I since the decision-makers have no external interests and are personally well insulated from fluctuations in demand for their input.

While real-world institutions are always more complex than theoretical models, it is worth noting that the British and U.S. industrial relations systems, for instance, have important features which are consistent with the institutional assumptions of Model I. The trade unions are certified to represent employees in a given firm and bargaining is company specific. Labor is usually supplied by the union and (re-) hired by the employer on the basis of seniority, which in turn leads to the creation of a well defined hierarchical structure. The employee with median seniority (median voter) is unlikely to be laid off but likely to vote for the union leadership, which negotiates the highest wage increases.

The Western European systems of participation by labor, management, and shareholders are also company specific. In a German firm, for instance, employee representatives to the board of directors and works council represent the interest of employees in the given enterprise. ${ }^{8}$ Since well-defined policies exist for hiring, layoffs, and discharges, and since the works council codetermines the wage and employment decisions of the company, one could argue that Model I applies in this setting as well. However, there are also important institutional features which might make Model II of the next section more applicable.

Finally, the Yugoslav labor-managed enterprises, as well as their semiautonomous subunits known as basic organizations of associated labor (BOALS), are widely believed to act largely in their own self-interest. While this does not necessarily make Model I the appropriate analytical tool, virtually all the existing theoretical literature on labor-managed firms represents a limiting (one party) case of this model.

Model I takes the total utility functions of labor $U_{L}^{T}$, management $U_{M}^{T}$, and shareholders $U_{S}^{T}$ to be

$$
\begin{aligned}
U_{L}^{T} & =W, \\
U_{M}^{T} & =m, \\
U_{S}^{T} & =r,
\end{aligned}
$$

where $W, m$, and $r$ are, respectively, the average wage, ${ }^{9}$ managerial salary, and rate of return on capital that the firm actually pays. Each group is also

\footnotetext{
${ }^{8}$ It is usually observed that these representatives represent all employees of the firm and not just the unionized ones. Here it must be stressed that they represent only employees of the firm and not other workers, whether unionized or not.

${ }^{9}$ The term average wage refers to the average income per unit of time that a worker receives from the firm.
} 
assumed to have some minimum unit price or threat point below which it would not supply any of its input to the firm. Denote these prices $W^{a}, m^{a}$, and $r^{a}$, respectively. They are usually taken to be the input prices that each party can obtain in the best alternative (market) employment of its input. The corresponding incremental utility functions are

$$
\begin{aligned}
& U_{L}=W-W^{a}, \\
& U_{M}=m-m^{a}, \\
& U_{S}=r-r^{a} .
\end{aligned}
$$

In accordance with (4), the three parties are seen to act as if maximizing ${ }^{10}$

$$
U=\left(W-W^{a}\right)^{\gamma_{L}}\left(m-m^{a}\right)^{\gamma_{M}}\left(r-r^{a}\right)^{\gamma_{S}}
$$

subject to the constraint that total expenditures equal total revenues: $\sum_{i=1}^{k} P_{i} Q_{i}-W L-m M-r K-\sum_{j=1}^{n} Z_{j}^{a} X_{j}-\pi_{0}=0$, where $P_{i}$ is the price of product $Q_{i}(i=1, \ldots, k), Z_{j}^{a}$ is the market price of input $X_{j}(j=1, \ldots, n)$ and $\pi_{0} \geqslant 0$ is an ex ante determined fixed charge (cost or tax) which goes to some party not involved in the bargaining process (e.g., government).

The term $\pi_{0}$ plays an important part in the theory of a participatory (including labor-managed) firm embedded in Model I. In particular, there are values of $\pi_{0}$ which, when coupled with certain technologies, lead to a nonexistence problem. In fact a maximum exists only in the following cases: (a) when $\pi_{0}>0$ and the production function $f$ exhibits decreasing returns to scale, (b) when $\pi_{0}=0$ and $f$ exhibits constant returns to scale, and (c) when $\pi_{0} \geqslant 0$ and the production function exhibits first increasing and then decreasing returns to scale.

In this paper it is assumed that $\pi_{0}>0$ and that the production set is strictly convex (case (a)). The Lagrangean expression for this problem can be written in a logarithmic form as

$$
\begin{aligned}
\mathscr{L}= & \gamma_{L} \ln \left(W-W^{a}\right)+\gamma_{M} \ln \left(m-m^{a}\right)+\gamma_{S} \ln \left(r-r^{a}\right) \\
& +\lambda_{1}\left(\sum_{i=1}^{k} P_{i} Q_{i}-W L-m M-r K-\sum_{j=1}^{n} Z_{j}^{a} X_{j}-\pi_{0}\right) \\
& +\lambda_{2} f\left(Q_{1}, \ldots, Q_{k}, L, M, K, X_{1}, \ldots, X_{n}\right),
\end{aligned}
$$

${ }^{10}$ The linear utility functions in (6) implicitly assume risk neutrality. It is clear, however, that the relative bargaining powers $\gamma_{1}, \gamma_{M}$, and $\gamma_{s}$ may be viewed as stemming from differential degrees of risk aversion of the three parties within the standard Nash $(1950,1953)$ model. Equations (7) can be viewed as the usual Nash maximand $U=U_{L} U_{M} U_{S}$ where $U_{L}=\left(W-W^{a}\right)^{\gamma_{L}}, U_{M}=\left(m-m^{a}\right)^{\gamma_{M}}$, and $U_{S}=\left(r-r^{a}\right)^{\gamma_{S}}$. In this case $\gamma_{L}, \gamma_{M}$, and $\gamma_{S}$ would be parameters of relative risk aversion reflecting the risk attitude of each party relative to others. The same interpretation of $\gamma_{L}, \gamma_{M}$, and $\gamma_{S}$ would not hold, however, if the utility functions in (6) reflected risk aversion directly (see Svejnar, 1980). 
with $\lambda_{1}$ and $\lambda_{2}$ being the Lagrange multipliers. The first-order conditions lead to the following results ${ }^{11}$ :

$$
\begin{array}{rlrl}
P_{i} \frac{\partial Q_{i}}{\partial L}=W=W^{a}+\gamma_{L} \frac{\pi}{L}, & i=1, \ldots, k, \\
P_{i} \frac{\partial Q_{i}}{\partial M}=m=m^{a}+\gamma_{M} \frac{\pi}{M}, & i=1, \ldots, k, \\
P_{i} \frac{\partial Q_{i}}{\partial K}=r=r^{a}+\gamma_{s} \frac{\pi}{K}, & i=1, \ldots, k, \\
P_{i} \frac{\partial Q_{i}}{\partial X_{j}}=Z_{j}^{a}, & i=1, \ldots, k, \\
j=1, \ldots, n,
\end{array}
$$

with $\pi=\sum_{i=1}^{k} P_{i} Q_{i}-W^{a} L-m^{a} M-r^{a} K-\sum_{j=1}^{n} Z_{j}^{a} X_{j}-\pi_{0}$.

Conditions (9)-(12) dictate that all resources ought to be hired so that the value of the marginal product of each input with respect to each output equals the input price paid by the firm. There is a crucial difference, however, between conditions (9)-(11) and condition (12). The latter implies that inputs $X_{j}(j=1, \ldots, n)$, whose owners do not participate in decisionmaking, are hired to the point where their values of the marginal product equal the market prices $Z_{j}^{a}(j=1, \ldots, n)$ of these inputs. In contrast, the optimal levels of $L, M$, and $K$ are given by the equality of the respective values of the marginal product and prices $W, m$, and $r$, which are determined within the firm. Each of these input prices $W, m$, and $r$ equals its corresponding best alternative (market) price $W^{a}, m^{a}$, and $r^{a}$, plus a share of the net profit $\pi$ per unit of input. The profit share is determined by the relative power of the party which supplies the input and $\pi$ is defined as revenues minus costs, with all inputs valued at the market prices.

Clearly, if $\pi=0$ there is no room for bargaining and therefore, $W=W^{a}$, $m=m^{a}, r=r^{a}$ and the allocational rule is identical for all the inputs. In this case the participatory firm allocates all resources in the same way as a traditional profit maximizing firm sharing the same technological and market conditions. ${ }^{12}$ As a result, the optimal behavior of a participatory firm is also efficient from the society's point of view when $\pi=0$.

"For the sake of simplicity product prices $P_{i}(i=1, \ldots, k)$ are taken as exogenous. If the firm has control over some $P_{i}$ then the results equate input prices with the marginal revenue products rather than values of marginal product. For example, equation (9), would then be $P_{i}\left(1+1 / \eta_{i}\right)\left(\partial Q_{i} / \partial L\right)=W=W^{a}+\gamma_{L}(\pi / L)$ with $\eta_{i}=\partial \ln Q_{i} / \partial \ln P_{i}$.

${ }_{12}$ This implies that the value of $\pi_{0}$ would be the same for the profit maximizing firm. The identity between the two types of firms follows from the fact that both maximize profit. The participatory firm in addition, determines how to divide the profit. When $\pi=0$ the participatory firm attains the maximum $U$ at $U=0$ in (7) and the profit maximizing firm maximizes profit at $\pi=0$. With identical technical and market conditions, the firms then behave identically in terms of factor allocation and the scale of operations. 
The interesting case arises when $\pi>0 .{ }^{13}$ Given $\gamma_{L}, \gamma_{M}, \gamma_{S}>0$, the participatory firm pays inputs $L, M$, and $K$ prices $W>W^{a}, m>m^{a}$, and $r>r^{a}$, respectively, while inputs $X_{j}(j=1, \ldots, n)$ are still paid their respective market prices $Z_{j}^{a}(j=1, \ldots, n)$. In contrast, a traditional cost minimizing firm, producing the same $Q_{i}(i=1, \ldots, k)$ and facing identical technological and market conditions, would still pay $L, M$, and $K$ the market prices $W^{a}, m^{a}$, and $r^{a}$, respectively, and allocate resources accordingly. Similarly, a technologically identical profit maximizing firm, while producing different levels of $Q_{i}(i=1, \ldots, k)$ at $\pi>0$, would still allocate resources so that the value of the marginal product of each input with respect to each output equals the market price of the given input.

At $\pi>0$, the optimum of the participatory firm does not coincide with the socially optimal factor allocation. Workers, managers, and shareholders, who supply their resources elsewhere at $W^{a}, m^{a}$, and $r^{a}$, respectively, would find it worthwhile to relocate into the participatory firms (sectors) where $W$, $m$, and $r$ are paid. It is against the interests of the incumbents in these firms, however, to admit the newcomers as full-fledged members of the respective parties. ${ }^{14}$ Hence, unless the incoming workers, managers, and shareholders are able to establish new firms and drive $\pi$ down to $\pi=0$ the misallocation of resources will persist.

Unfortunately, in the general case at hand, it is impossible to determine unambigously the nature of resource misallocation when $\pi>0$. It is likely that the participatory firm will use less $L, M$, and $K$ than the corresponding cost minimizing and profit maximizing firms. This claim cannot be rigorously substantiated, however, without knowing the degree of competitiveness and complementarity among the $(k+3+n)$ outputs and inputs. Assuming the relative $P_{i}$ 's and $Z_{j}^{a}$ 's, respectively, are constant, the general allocational problem reduces to that of one output $Q$ and four inputs $L, M, K$, and $X$. In the absence of Hicksian regression between $Q$ and every one of the three inputs $L, M$, and $K$, the conditions $W>W^{a}, m>m^{a}$, and $r>r^{a}$ imply that the participatory firm will produce smaller $Q$ than the corresponding profit maximizing firm. Since regression is a rare event, this outcome is likely. In terms of factor allocation, if $X$ is competitive to $L, M$, and $K$, then at least one of the $L / X, M / X$, and $K / X$ ratios will be lower ${ }^{15}$ in

${ }^{13}$ The case $\pi<0$ is irrelevant because at least one of the parties would not be able to obtain the market price for its input. A no-trade situation would result and the firm would go out of business.

${ }^{14}$ It would be in the incumbents" interest to hire the resources of these newcomers at $W^{a}$, $m^{a}$, and $r^{a}$ in the same way as they hire the other inputs $X_{j}$ at $Z_{j}^{a}(j=1, \ldots, n)$. If socially feasible, such an arrangement would lead to allocational (though not distributional) results of Model II.

${ }^{15}$ If $L, M$, and $K$ are complementary or not highly competitive along themselves, then all the $L / X, M / X$, and $K / X$ ratios will be lower in the participatory firm than in its profit maximizing counterpart. See Hicks (1946, pp. 92-98). 
the participatory firm than in the corresponding profit maximizing firm. In order to obtain more definite results, further restrictions can be imposed along the lines of separability, the number of parties and/or the number of inputs. Three sets of results, obtained along these lines, are reported in the Appendix.

Since the objective function depends on the relative powers $\gamma_{L}, \gamma_{M}$, and $\gamma_{S}$, it is essential to understand the role that $\gamma_{L}, \gamma_{M}$, and $\gamma_{S}$ play in Model I. Equations (9)-(11) simplify to

$$
\begin{aligned}
& \frac{W-W^{a}}{m-m^{a}}=\frac{\gamma_{L}}{\gamma_{M}} \frac{M}{L} \Rightarrow \ln \left(\frac{W-W^{a}}{m-m^{a}}\right)=\ln \left(\frac{\gamma_{L}}{\gamma_{M}}\right)+\ln \left(\frac{M}{L}\right), \\
& \frac{W-W^{a}}{r-r^{a}}=\frac{\gamma_{L}}{\gamma_{S}} \frac{K}{L} \Rightarrow \ln \left(\frac{W-W^{a}}{r-r^{a}}\right)=\ln \left(\frac{\gamma_{L}}{\gamma_{S}}\right)+\ln \left(\frac{K}{L}\right) .
\end{aligned}
$$

Differentiating (13) with respect to $\left(\gamma_{L} / \gamma_{M}\right)$ and (14) with respect to $\left(\gamma_{L} / \gamma_{S}\right)$ leads to:

$$
\begin{aligned}
& \begin{aligned}
\frac{d\left(\frac{W-W^{a}}{m-m^{a}}\right)}{d\left(\gamma_{L} / \gamma_{M}\right)} & =\frac{M}{L}+\frac{\gamma_{L}}{\gamma_{M}} \frac{d(M / L)}{d\left(\gamma_{L} / \gamma_{M}\right)} \Rightarrow \frac{d \ln \left(\frac{W-W^{a}}{m-m^{a}}\right)}{d \ln \left(\gamma_{L} / \gamma_{M}\right)} \\
& =1+\frac{d \ln (M / L)}{d \ln \left(\gamma_{L} / \gamma_{M}\right)}, \\
\frac{d\left(\frac{W-W^{a}}{r-r^{a}}\right)}{d\left(\gamma_{L} / \gamma_{M}\right)} & =\frac{K}{L}+\frac{\gamma_{L}}{\gamma_{S}} \frac{d(K / L)}{d\left(\gamma_{L} / \gamma_{S}\right)} \Rightarrow \frac{d \ln \left(\frac{W-W^{a}}{r-r^{a}}\right)}{d \ln \left(\gamma_{L} / \gamma_{S}\right)} \\
& =1+\frac{d \ln (K / L)}{d \ln \left(\gamma_{L} / \gamma_{S}\right)} .
\end{aligned}
\end{aligned}
$$

Equations (15) and (16) indicate that changes in relative powers of the parties will result in redistribution of the net profit among the parties in two ways. The first redistribution represents an input-constant effect, due purely to the changes in the relative powers. In (15) and (16), with labor's power rising at the expense of the power of management and shareholders, this distributional effect equals $M / L$ and $K / L$, respectively. ${ }^{16}$ The last terms on the right-hand sides of (15) and (16) represent redistributions of income, which the changing $\gamma_{L}, \gamma_{M}$, and $\gamma_{S}$ bring about through reallocation of $L, M$, and $K$. The total effect on profit distribution of changing $\gamma_{L}, \gamma_{M}$, and $\gamma_{S}$ can

${ }^{16}$ The effect in (15) and (16) equals $M / L$ and $K / L$, respectively, because the transfer is denominated in the unit input price of each input. When evaluated in percentage terms it is equal to one. 
thus be decomposed into the input-constant direct effect and the indirect effect, which is due to changes in the input ratios. Hence, $\gamma_{L}, \gamma_{M}$, and $\gamma_{S}$ have not only distributional but also allocational significance in Model $\mathrm{I}$.

While the input-constant direct effect is positive and clearly defined, the sign of the indirect "input-substitution" effect is not a priori obvious. Model I postulates that the parties maximize their respective unit prices, regardless of what happens to the employment of their inputs. An increase in the relative power of a given party will therefore raise the relative price of its input but it may increase or decrease the relative use of the input by the firm. More light can be thrown on this issue through the following approach.

Let $W(m, r)=\max \{W \mid L, M, K, X, P Q(L, M, K, X)-W L-m M-r K$ $\left.-Z^{a} X-\pi_{0} \geqslant 0\right\}$. Since $Q$ is strictly concave in $L, M, K$, and $X$, there exist $L, M, K, X>0$ such that $P Q(L, M, K, X)-W(m, r) L-m M-r K-Z^{a} X-$ $\pi_{0} \leqslant 0$.

Proposition 1. $W(m, r)$ is decreasing in $m$ and $r$.

Proof. Let $\pi^{*}=P Q(L, M, K, X)-W(m, r) L-m M-r K-Z^{a} X-\pi_{0} \geqslant 0$. For given $L, M, K$, and $X$ the minimum $\pi^{*}=0$, which corresponds to $W(m, r)$, satisfies

$$
\begin{aligned}
& \frac{\partial \pi^{*}}{\partial m}=-\frac{\partial W}{\partial m} L-M=0, \\
& \frac{\partial \pi^{*}}{\partial r}=-\frac{\partial W}{\partial r} L-K=0 .
\end{aligned}
$$

Rearranging the terms in these two equations yields

$$
\begin{aligned}
& \frac{\partial W}{\partial m}=-\frac{M}{L}<0, \\
& \frac{\partial W}{\partial r}=-\frac{K}{L}<0 .
\end{aligned}
$$

Q.E.D.

Proposition 2. $W(m, r)$ is concave.

Proof. Let $[W(m, r), m, r, L, M, K, X]$ and $\left[W\left(m^{\prime}, r^{\prime}\right), m^{\prime}, r^{\prime}, L^{\prime}, M^{\prime}\right.$, $K^{\prime}, X^{\prime} \mid$ be two $\pi^{*}=0$ combinations of input prices and inputs. That is, let

$$
\begin{aligned}
& \pi^{*}=P Q(L, M, K, X)-W(m, r) L-m M-r K-Z^{a} X-\pi_{0}=0, \\
& \pi^{*}=P Q\left(L^{\prime}, M^{\prime}, K^{\prime}, X^{\prime}\right)-W\left(m^{\prime}, r^{\prime}\right) L^{\prime}-m^{\prime} M^{\prime}-r^{\prime} K^{\prime}-Z^{a} X^{\prime}-\pi_{0}=0 .
\end{aligned}
$$


Moreover, let $m^{\prime \prime}=t m+(1-t) m^{\prime}$ and $r^{\prime \prime}=\operatorname{tr}+(1-t) r^{\prime}$ with $0 \leqslant t \leqslant 1$. Now for some $L^{\prime \prime}, M^{\prime \prime}, K^{\prime \prime}, X^{\prime \prime}, m^{\prime \prime}$, and $r^{\prime \prime}$ there is $W\left(m^{\prime \prime}, r^{\prime \prime}\right)$, which minimizes

$$
\pi^{*}=P Q\left(L^{\prime \prime}, M^{\prime \prime}, K^{\prime \prime}, X^{\prime \prime}\right)-W\left(m^{\prime \prime}, r^{\prime \prime}\right) L^{\prime \prime}-m^{\prime \prime} M^{\prime \prime}-r^{\prime \prime} K^{\prime \prime}-Z^{a} X^{\prime \prime}-\pi_{0} .
$$

Since neither $m$ and $r$ nor $m^{\prime}$ and $r^{\prime}$ necessarily represent the prices of $M$ and $K$ that minimize $\pi^{*}$ at $L^{\prime \prime}, M^{\prime \prime}, K^{\prime \prime}$, and $X^{\prime \prime}$, it follows that

$$
\begin{aligned}
& P Q\left(L^{\prime \prime}, M^{\prime \prime}, K^{\prime \prime}, X^{\prime \prime}\right)-W\left(m^{\prime \prime}, r^{\prime \prime}\right) L^{\prime \prime}-m^{\prime \prime} M^{\prime \prime}-r^{\prime \prime} K^{\prime \prime}-Z^{a} X^{\prime \prime}-\pi_{0} \\
& \quad \leqslant P Q\left(L^{\prime \prime}, M^{\prime \prime}, K^{\prime \prime}, X^{\prime \prime}\right)-W(m, r) L^{\prime \prime}-m M^{\prime \prime}-r K^{\prime \prime}-Z^{a} X^{\prime \prime}-\pi_{0}
\end{aligned}
$$

and

$$
\begin{aligned}
& P Q\left(L^{\prime \prime}, M^{\prime \prime}, K^{\prime \prime}, X^{\prime \prime}\right)-W\left(m^{\prime \prime}, r^{\prime \prime}\right) L^{\prime \prime}-m^{\prime \prime} M^{\prime \prime}-r^{\prime \prime} K^{\prime \prime}-Z^{a} X^{\prime \prime}-\pi_{0} \\
& \quad \leqslant P Q\left(L^{\prime \prime}, M^{\prime \prime}, K^{\prime \prime}, X^{\prime \prime}\right)-W\left(m^{\prime}, r^{\prime}\right) L^{\prime \prime}-m^{\prime} M^{\prime \prime}-r^{\prime} K^{\prime \prime}-Z^{a} X^{\prime \prime}-\pi_{0} .
\end{aligned}
$$

As a result,

$$
\begin{aligned}
P Q\left(L^{\prime \prime},\right. & \left.M^{\prime \prime}, K^{\prime \prime}, X^{\prime \prime}\right)-W\left(m^{\prime \prime}, r^{\prime \prime}\right) L^{\prime \prime}-m^{\prime \prime} M^{\prime \prime}-r^{\prime \prime} K^{\prime \prime}-Z^{a} X^{\prime \prime}-\pi_{0} \\
\leqslant & P Q\left(L^{\prime \prime}, M^{\prime \prime}, K^{\prime \prime}, X^{\prime \prime}\right)-\left[t W(m, r)+(1-t) W\left(m^{\prime}, r^{\prime}\right)\right] L^{\prime \prime} \\
& -m^{\prime \prime} M-r^{\prime \prime} K^{\prime \prime}-Z^{a} X^{\prime \prime}-\pi_{0},
\end{aligned}
$$

which leads to

$$
W\left(m^{\prime \prime}, r^{\prime \prime}\right) \geqslant t W(m, r)+(1-t) W\left(m^{\prime}, r^{\prime}\right)
$$

Propositions 1 and 2 provide enough information to show that it is unlikely that the sign of the indirect "input-substitution" effect of $\gamma_{L}, \gamma_{M}$, and $\gamma_{s}$ on the distribution of the net profit can be analytically determined. Substituting (17) into (15) and (18) into (16) leads to

$$
\begin{aligned}
& \frac{d\left(\frac{W-W^{a}}{m-m^{a}}\right)}{d\left(\gamma_{L} / \gamma_{M}\right)}=-W_{1}+\frac{\gamma_{L}}{\gamma_{M}} \frac{\partial\left(-W_{1}\right)}{\partial\left(\gamma_{L} / \gamma_{M}\right)}, \\
& \frac{d\left(\frac{W-W^{a}}{r-r^{a}}\right)}{d\left(\gamma_{L} / \gamma_{S}\right)}=-W_{2}+\frac{\gamma_{L}}{\gamma_{S}} \frac{\partial\left(-W_{2}\right)}{\partial\left(\gamma_{L} / \gamma_{S}\right)},
\end{aligned}
$$

where $W_{1}=\partial W / \partial m$ and $W_{2}=\partial W / \partial r$. The first terms on the right-hand side of (19) and (20) are positive by Proposition 1.

To see that the last terms are not a priori determinate, write $m=m$ $\left(\gamma_{L}, \gamma_{M}, \gamma_{S}\right)=m\left(\gamma_{L} / \gamma_{M}, \gamma_{L} / \gamma_{S}\right)$ as $m\left(\zeta_{M}, \zeta_{S}\right)$ and $r=r\left(\gamma_{L}, \gamma_{M}, \gamma_{S}\right)=$ 
$r\left(\gamma_{L} / \gamma_{M}, \gamma_{L} / \gamma_{S}\right)$ as $r\left(\zeta_{M}, \zeta_{S}\right)$, with $\zeta_{M}=\gamma_{L} / \gamma_{M}$ and $\zeta_{S}=\gamma_{L} / \gamma_{S}$. The derivatives in the last terms of (19) and (20) then become $\partial\left[-W_{1}(m, r)\right] / \partial \zeta_{M}=$ $-W_{11}(m, r)\left(\partial m / \partial \zeta_{M}\right)-W_{12}(m, r)\left(\partial r / \partial \zeta_{M}\right) \quad$ and $\quad \partial\left[-W_{2}(m, r)\right] / \partial \zeta_{S}=$ $-W_{21}(m, r)\left(\partial m / \partial \zeta_{S}\right)-W_{22}(m, r)\left(\partial r / \partial \zeta_{S}\right)$, respectively. Now, while $W_{11}$ and $W_{22}$ are negative by Proposition 2, the signs of $W_{12}=W_{21}, \partial m / \partial \zeta_{M}, \partial r / \partial \zeta_{M}$, $\partial m / \partial \zeta_{S}$, and $\partial r / \partial \zeta_{S}$ cannot be determined in general. Depending on specific circumstances, the total effect of a rise in the power of a given party may therefore exceed or fall short of the direct effect alone. However, since labor's objective is to increase $W-W^{a}$ and since $\partial \zeta_{M}, \partial \zeta_{S}$ represent an increase in labor's power, it is expected that the overall effect in (19) and (20) is positive.

\section{MODEL II}

Model II assumes that the parties involved in bargaining have constituencies or interests which transcend the individual firm. In contrast to Model I, this model describes a situation where the total quantities of inputs supplied (represented) by the bargainers may not be employed in the single firm. Each party therefore pursues objectives relating to the income of its relatively "large" constituency rather than merely the fraction of its constituency employed in a given firm.

More formally, Model II deals with the situation where there are large fixed constituencies of $\bar{L}, \bar{K}$, and $\bar{M}$ whose expected income is to be maximized by their representatives (bargainers) irrespective of the fractions of $\bar{L}, \bar{K}$, and $\bar{M}$ that are employed in the particular firm. An important point is that the given constituency of workers $\bar{L}$, for example, need not be the entire group of workers in the country, but merely some group larger than the workers employed by the firm.

There are numerous examples of institutions which contain elements that fit Model II. In contrast to the Anglo-American trade unions, unions in many Western European countries have broad constituencies because of the industry, regional and even nation-wide systems of collective bargaining between trade union federations and employer organizations. Moreover, in many of these countries, a well defined rule such as seniority is not used as the sole criterion for lay-offs and rehiring. ${ }^{17}$ Consequently, one can expect that there is a strong incentive for Western European trade unions to

\footnotetext{
${ }^{17}$ It is often customary to decrease the number of hours worked by all workers in the periods of low demand rather than laying-off workers. The social situation of the workers and their families is often among factors considered in determining who is layed-off and who is rehired in a given firm. As a result, it is much harder to identify a well-defined hierarchy (median voter) in a given firm which would determine the union goals.
} 
maximize the expected wage of all their constituents rather than merely the wage of those remaining with a given firm. ${ }^{18}$

While the Western European participatory schemes, such as works councils and participation on company boards, are primarily company specific, it should be noted that trade unions often play a substantial role in creating these schemes and exert considerable influence in the selection of worker representatives and in the formulation of the economic policies of these bodies. The interesting empirical question is whether the unions' influence over these representative bodies is sufficient to propagate the broader perspective of Model II or whether the more narrow (companyspecific) goals of Model I prevail.

As was pointed out at the start of Section III, the behavior of the Yugoslav labor-managed enterprises has been modelled by numerous authors in the spirit of Model I. While the highly publicized autonomy enjoyed by the Yugoslav firms seemingly supports this classification, it is of interest that in his detailed institutional analysis of the Yugoslav system, Vanck (1972) did not find support for the "Ward model" in terms of resource allocation. ${ }^{19}$ It must also be remembered that the Yugoslav firms operate in an environment in which government-sponsored trade unions, various government agencies, and regional as well as community organizations exert influence over the firms' policies. In view of the severe unemployment problem confronting the Yugoslav economy, the influence of these parties is likely to reflect the features of Model II. Whether the broader perspectives of these parties outweigh the presumed self-centered orientation of the workers insulated within a given firm is again an empirical issue.

In accordance with the institutional features described here, Model II is formulated so that each party maximizes the expected income of its constituents. The total utility functions of labor, management, and shareholders may, hence, be defined as

$$
\begin{aligned}
& U_{L}^{T}=W \frac{L}{\bar{L}}+\left(1-\frac{L}{\bar{L}}\right) W^{a} \\
& U_{M}^{T}=m \frac{M}{\bar{M}}+\left(1-\frac{M}{\bar{M}}\right) m^{a}, \\
& U_{S}^{T}=r \frac{K}{\bar{K}}+\left(1-\frac{K}{\bar{K}}\right) r^{a},
\end{aligned}
$$

${ }^{18}$ As a result of their overwhelming economic and political power, many Western European unions also feel at least partially reponsible for their country's overall economic situation which may even transcend their industry- or sector-specific interests.

${ }^{19}$ The Ward (1958) model of a labor-managed firm was later extended by Domar (1966), Jaroslav Vanek (1970), and others. It corresponds to Model I in this paper with $\gamma_{M}=\gamma_{S}=0$. 
where $\bar{L}, \bar{M}$, and $\bar{K}$ are the total amounts of labor, managerial input and capital supplied by the three parties, and $L, M$, and $K$ are the levels of these inputs that are employed in the given firm at the negotiated prices $W, m$, and $r$, respectively. By assumption $L / \bar{L}<1, M / \bar{M}<1$, and $K / \bar{K}<1$. The fractions $1-(L / \bar{L}), 1-(M / \bar{M})$, and $1-(K / \bar{K})$ are the proportions of the three inputs that are employed at the respective best alternative (market) prices $W^{a}, m^{a}$ and $r^{a} .{ }^{20}$ Taking, $W^{a}, m^{a}$ and $r^{a}$ as the threat points, the incremental utility functions are

$$
\begin{aligned}
& U_{L}=\left(W-W^{a}\right) \frac{L}{\bar{L}}, \\
& U_{M}=\left(m-m^{a}\right) \frac{M}{\bar{M}} \\
& U_{S}=\left(r-r^{a}\right) \frac{K}{\bar{K}} .
\end{aligned}
$$

The Lagrangean for this maximization problem may be written in a logarithmic form as:

$$
\begin{aligned}
\mathscr{P}^{\prime}= & \gamma_{L}\left[\ln \left(W-W^{a}\right)+\ln L-\ln \bar{L}\right]+\gamma_{M}\left[\ln \left(m-m^{a}\right)+\ln M-\ln \bar{M}\right] \\
& +\gamma_{S}\left[\ln \left(r-r^{a}\right)+\ln K-\ln \bar{K}\right] \\
& +\lambda_{1}^{\prime}\left(\sum_{i=1}^{k} P_{i} Q_{i}-W L-m M-r K-\sum_{j=1}^{n} Z_{j}^{a} X_{j}-\pi_{0}\right) \\
& +\lambda_{2}^{\prime} f\left(Q_{1}, \ldots, Q_{k}, L, M, K, X_{1}, \ldots, X_{n}\right),
\end{aligned}
$$

where $\lambda_{1}^{\prime}$ and $\lambda_{2}^{\prime}$ are the Lagrange multipliers. The corresponding first-order conditions imply

$$
\begin{aligned}
& P_{i} \frac{\partial Q_{i}}{\partial L}=W^{a}, \quad i=1, \ldots, k, \\
& P_{i} \frac{\partial Q_{i}}{\partial M}=m^{a}, \quad i=1, \ldots, k, \\
& P_{i} \frac{\partial Q_{i}}{\partial K}=r^{a}, \quad i=1, \ldots, k,
\end{aligned}
$$

${ }^{20}$ Alternatively, one can assume that each party maximizes the income bill that it can generate with the input it supplies. The total utility function are then $U_{L}^{T}=W L+(\bar{L}-L) W^{a}$. $U_{M}^{T}=m M+(\bar{M}-M) m^{a}$, and $U_{S}^{T}=r K+(\bar{K}-K) r^{a}$. With $W^{a} \bar{L}, m^{a} \bar{M}$, and $r^{a} \bar{K}$ being the resistance points, the incremental utility functions become $U_{L}=\left(W-W^{a}\right) I$, $U_{M}=\left(m-m^{a}\right) M$ and $U_{S}=\left(r-r^{a}\right) K$. The results of this approach are identical with those of Model II in the main text. 


$$
\begin{aligned}
P_{i} \frac{\partial Q_{i}}{\partial X_{j}}=Z_{j}^{a}, \quad i=1, \ldots, k ; j=1, \ldots, n, & \\
W & =W^{a}+\gamma_{L} \frac{\pi}{L}, \\
m & =m^{a}+\gamma_{M} \frac{\pi}{M}, \\
r & =r^{a}+\gamma_{S} \frac{\pi}{K},
\end{aligned}
$$

where $\pi=\sum_{i=1}^{k} P_{i} Q_{i}-W^{a} L-m^{a} M-r^{a} K-\sum_{j=1}^{n} Z_{j}^{a} X_{j}-\pi_{0}$ as in Model I. Equations (25)-(27) give expressions for $W, m$, and $r$ that are identical with those in Model I (Eqs. (9)-(11)). Each party sccures an input price which is equal to the best alternative (market) price plus a share in the net profit $\pi$ per unit of input employed. The share is again determined by the relative power of the given party. It is also evident, however, that while the expressions for $W, m$, and $r$ coincide in the two models, actual $W, m$, and $r$ may differ because $L, M$, and $K$ are determined by different allocational rules in the two models. ${ }^{21}$ Equations (21)-(24) imply that in Model II all resources are hired so that the marginal value product of each input with respect to each output is equated to the best alternative (market) price of that input. Thus rule applies to all inputs, irrespective of whether their owners codetermine the company policy or not.

Therefore, in contrast to Model $\mathbf{I}$, decisions concerning optimal resource allocation are separate from input price determination in Model II. In fact, the decision-making process of Model II may be thought of as proceeding in two stages. First, resources are allocated according to (21)-(24). The resulting optimal values of $L, M, K, X_{j}(j=1, \ldots, n)$ and $Q_{i}(i=1, \ldots, k)$, together with the parametrically given values of $W^{a}, m^{a}, r^{a}, Z_{j}^{a}(j=1, \ldots, n)$ and $P_{i}(i=1, \ldots, k)$, determine the net profit $\pi$. If $\pi>0$, then in the second stage of decision-making the parties involved distribute $\pi$ among themselves in proportion to their relative powers.

Unlike Model I, the relative powers $\gamma_{L}, \gamma_{M}$, and $\gamma_{S}$ have no allocational role in Model II. They merely determine the distribution of the net profit, when $\pi>0$. Rearrange (25)-(27) as

$$
\begin{aligned}
& \frac{W-W^{a}}{m-m^{a}}=\frac{\gamma_{L}}{\gamma_{M}} \frac{M}{L}, \\
& \frac{W-W^{a}}{r-r^{a}}=\frac{\gamma_{L}}{\gamma_{S}} \frac{K}{L} .
\end{aligned}
$$

${ }^{21}$ In particular, the partial derivatives in (21)-(24) are evaluated at different points than in (9)-(12) when $\pi>0$. 
Equations (28) and (29) look identical with (13) and (16) of Model I. However, there is a major distinction between the two sets of conditions and it lies in the fact that $L, M$, and $K$ are determined independently of $\gamma_{L}, \gamma_{M}$, and $\gamma_{S}$ in Model II. Consequently, any variation in the relative powers will be reflected directly in the relative incremental incomes of the three parties. No indirect "input-substitution" effect exists in Model II. The direct effect is the total effect

$$
\begin{aligned}
& \frac{d\left(\frac{W-W^{a}}{m-m^{a}}\right)}{d\left(\gamma_{L} / \gamma_{M}\right)}=\frac{M}{L} \Rightarrow \frac{d \ln \left(\frac{W-W^{a}}{m-m^{a}}\right)}{d \ln \left(\gamma_{L} / \gamma_{M}\right)}=1, \\
& \frac{d\left(\frac{W-W^{a}}{r-r^{a}}\right)}{d\left(\gamma_{L} / \gamma_{S}\right)}=\frac{K}{L} \Rightarrow \frac{d \ln \left(\frac{W-W^{a}}{r-r^{a}}\right)}{d \ln \left(\gamma_{L} / \gamma_{S}\right)}=1 .
\end{aligned}
$$

Finally, it is worth noting that while the term $\pi_{0}$ has been included in the analysis, it plays no crucial part in Model II. A maximum exists so long as the production function does not exhibit increasing returns to scale throughout.

\section{DisCuSSION OF THE RESULTS}

The foregoing analysis provides several important results:

(1) When the net profit $\pi$ is zero, participatory firms described in the two models behave identically, provided they share the same technology and market conditions. In this case the two firms are also indistinguishable from a traditional profit maximizing firm, which faces the same technological and market constraints. All three types of firms allocate resources efficiently.

(2) The interesting case arises when $\pi>0$. The participatory firm of Model II still behaves identically with the corresponding profit maximizing firm in terms of factor allocation and output supply. The only difference between them lies in the distribution of the net profit $\pi$. The participatory firm of Model I follows a different allocational rule, however, and while maximizing its objective function, it allocates resources inefficiently from the social point of view.

(3) The role of relative powers $\gamma_{L}, \gamma_{M}$, and $\gamma_{S}$ which the parties have over the firm's policy differs in the two models. While in Model II the relative powers influence only the distribution of $\pi$, in Model I they have allocational significance as well.

(4) In Model I the ex ante determined term $\pi_{0}$ (fixed cost or a lump sum tax) plays a crucial part in that some values of $\pi_{0}$, when coupled with 
certain technologies, lead to a non-existence problem. Model II generates no such problems. It merely requires non-increasing returns to scale for a maximum to exist.

The different conclusions that can be derived from the two models naturally raise the empirical question as to whether given institutions give rise to participatory firms of Model I or Model II. Since the two models were intentionally formulated so as to represent two polar cases of socioeconomic behavior, various hybrid and possibly more realistic cases could be envisioned, with some parties acting in the spirit of Model I and other of Model II. The existing literature on participatory and labormanaged firms employed models which are in the class of Model I. As a result, the inefficiencies associated with this model have become virtually synonymous with the presumed behavior of participatory and labor-managed firms. Various authors, including Ireland and Law (1978), have developed artificial schemes for correcting the perceived misallocation of resources in labor-managed firms and economies. Yet, as the institutional introductions to Models I and II indicate, there is enough casual empirical evidence to suggest that at least some of the existing institutional systems may contain participatory firms behaving according to Model II. Hence, while corrective schemes may be useful as policy tools vis a vis the participatory firms of Model I, it is first necessary to determine empirically whether the relevant institutions indeed foster the behavior implicit in that model.

Since (a) the two models present testable hypothesis and (b) very little empirical work has been done in this area, the next important step would clearly be to test the allocational and distributional effects of $\gamma_{L}, \gamma_{M}$, and $\gamma_{S}$. Using equations corresponding to (9) and (25) of this paper, Svejnar $(1977,1981)$ found positive wage effect associated with the introduction of certain forms of German codetermination. These empirical results are compatible with both Model I and II. In order to distinguish between the two models empirically, tests need to be formulated in terms of Eqs. (15)-(16) and (30)-(31).

\section{APPENDIX}

In order to derive more specific results about the behavior of a Model I participatory firm when $\pi>0$, restrictions need to be imposed either in terms of separability of the production function, the number of partics and/or the number of inputs. This appendix presents the results of three different sets of assumptions:

(a) Only labor and shareholders participate in decision-making and $L$ and $K$ are the only inputs. In this case the production function is 
$Q=Q(L, K)$. The first-order conditions are $P(\partial Q / \partial L)=W>W^{u}$ and $P(\partial Q / \partial K)=r>r^{a}$ at $\pi>0$, as compared to $P(\partial Q / \partial L)=W^{a}$ and $P(\partial Q / \partial K)=r^{a}$ at $\pi=0$. Hence, at $\pi>0$ both $L$ and $K$ command higher prices than they would receive in the corresponding profit maximizing firm. In the absence of Hicksian regression between $Q$ and both $L$ and $K$, this means that the participatory firm will operate at a lower level of output than its profit maximizing counterpart. For a homothetic production function it is also true that the $K / L$ ratio will be higher (lower) in the participatory firm than in the profit maximizing firm if $W / r>W^{a} / r^{a}\left(W / r<W^{a} / r^{a}\right)$. If the comparison is made between a participatory and a corresponding cost minimizing firm, then this condition holds even for a non-homothetic production function. As far as the absolute levels of $L$ and $K$ are concerned, the participatory firm will use less of both $L$ and $K$ than its profit maximizing counterpart, unless (i) $W$ and $r$ constitute very uneven increases from $W^{a}$ and $r^{a}$, and (ii) high competitiveness exists between $L$ and $K$. If (i) and (ii) hold, then the factor, whose price rises relatively less, might actually be used more than at $W^{a}$ and $r^{a}$; Hicks (1964, pp. 92-98).

(b) Labor, managers, and shareholders participate in decision-making $\left(\gamma_{L}, \gamma_{M}, \gamma_{S}>0\right), L, M, K$, and $X$ are used in production of $Q$, and the production function is additively separable in all its inputs- $Q=c(L)+$ $d(M)+e(K)+f(X)$. Since $P(\partial Q / \partial L)=P c^{\prime}(L)=W>W^{a}, \quad P(\partial Q / \partial M)=$ $P d^{\prime}(M)=m>m^{a}, \quad P(\partial Q / \partial K)=P e^{\prime}(K)=r>r^{a}, \quad$ and $P(\partial Q / \partial X)=$ $P f^{\prime}(X)=Z^{a}$ at $\pi>0$, it follows that the participatory firm will use less $L$, $M$, and $K$, and produce smaller $Q$ then the profit maximizing firm. Input $X$ will be used identically in both firms.

(c) Managers do not participate in decision-making $\left(\gamma_{M}=0\right), L, M, K$, and $X$ are used in production of $Q$, and the production function is pairwise additively separable as follows: $Q=f(L, K)+g(M, X)$. It this case, $P(\partial Q / \partial L)=f_{L}(L, K)=W>W^{a}, P(\partial Q / \partial K)=f_{K}(L, K)=r>r^{a}, P(\partial Q / \partial M)=$ $g_{M}(M, X)=m^{a}$, and $P(\partial Q / \partial X)=g_{X}(M, X)=Z^{a}$ at $\pi>0$. The levels of $L, K$, and $Q$ are determined by the same rules as in (a) above. In the absence of a regression between $Q$ and both $L$ and $K$, the participatory firm will operate at a lower $Q$ than the profit maximizing firm when $\pi>0$. If $f(L, K)$ is homothetic, then the $K / L$ ratio will be higher (lower) in the participatory firm than in the profit maximizing one as long as $W / r>$ $W^{a} / r^{a}\left(W / r<W^{a} / r^{a}\right)$. Finally, the participatory firm will use less $L$ and $K$ than its profit maximizing counterpart unless conditions (i) and (ii) of (a) prevail. Since $g(M, X)$ is independent of $f(L, K)$ and $g_{M}(M, X)=m^{a}$ and $g_{X}(M, X)=Z^{a}$, it follows that the utilization of $M$ and $X$ will be identical in both firms.

The three examples in this appendix demonstrate that definite results can be obtained for specific situations. Numerous other outcomes could, of 
course, be generated analogously from different technical and behavioral assumptions.

\section{REFERENCES}

Domar, E. D. (1966), The Soviet collective farm as a producer cooperative, Amer. Econ. Rev. 56, No. 4, September

Hicks, J. (1946), "Value and Capital," Oxford Univ. Press, London.

IRELAND, N. J. AND LAw, P. J. (1978), An incentive fund for labour mobility in the cooperative economy, Economica 45, No. 178, May.

KALAI, E. (1977), Nonsymmetric Nash solutions and replications of two-person bargaining, Internat. J. Game Theory 6.

NASH, J. F., JR. (1950), The bargaining problem, Econometrica 18, April.

NASH, J. F., JR. (1953), Two person cooperative games, Econometrica 21, January.

Roth, A. E. (1979), "Axiomatic Models of Bargaining," Lecture Notes in Economics and Mathematical Systems No. 170, Springer-Verlag, Berlin/New York/Heidelberg.

SCHREGLE, J. (1976), Workers' participation in decisions within undertakings, Internat. Labour Rev. 113, No. 1, January-February.

STEINHERR, A. (1977), On the efficiency of profit sharing and labor participation in management, Bell J. Econ. 8, No. 2, Autumn.

SvejNAR, J. (1977), "The Effect of Employee Participation in Management on Bargaining Power and Wages: A generalized Nash Solution and Econometric Evidence from Germany," Working Paper No. 106, Industrial Relations Section, Princeton University, Deccmber.

SvejnAR, J. (1980), "The Bargaining Problem with Variable Bargaining Powers," paper presented at the North-American Meetings of the Econometric Society, Denver, Colorado, September.

SveJNAR, J. (1981), Relative wage effects of unions, dictatorship, and codetermination, Rev. Econ. Statist. 63, No. 2, May.

SVEJNAR, J. (1982), Employee participation in management, bargaining power and wages. Europ. Econ. Rev. 17, No. 3.

VANEK, JAN (1972), "The Economics of Workers' Management," Allen \& Unwin, London.

VANEK, JAR. (1970), "The General Theory of Labor-Managed Market Economies," Cornell Univ. Press, Ithaca, New York.

WARD, B (1958), The firm if Illyria: Market syndicalism, Amer. Econ. Rev. 48, No. 4, September.

Windmuller, J. (1977), Industrial democracy and industrial relations, Ann. Amer. Acad. Pol. Social Sci. 431, May. 